\title{
Complications of cannulation of the ascending aorta for open heart surgery
}

\author{
FA YEK D. SALAMA ${ }^{1}$ and ARY BLESOVSK Y \\ Department of Cardiothoracic Surgery, Shotley Bridge General Hospital
}

In a series of 420 ascending aortic cannulations for cardiopulmonary bypass, major complications occurred in three patients. Aortic dissection occurred in one patient believed to be due to aortic cross-clamping. Avoidance of this manœuvre is suggested when the aorta is grossly atheromatous. Two patients developing false aneurysms due to mediastinal infection are described. Prevention of mediastinal infection and its treatment are discussed. The method of treatment of false aneurysms by deep hypothermia is described.

Cannulation of the ascending aorta for arterial return during cardiopulmonary bypass is a simple and convenient procedure which, in many units, is used in preference to cannulation of the femoral or iliac artery. The method is generally safe with a low complication rate.

\section{CASE REPORTS}

CASE 1 A 58-year-old woman had the mitral valve replaced in December 1967. The ascending aorta was dilated in the absence of aortic valve disease. Routine cannulation of the aorta was performed without difficulty.

During replacement of the mitral valve there was enough aortic incompetence to interfere with the procedure and the aorta had to be clamped three times. On releasing the clamp after the third period of clamping, the aortic reflux was considerably less than before, and shortly after this the electrocardiogram showed gross ischaemic changes and the heart beat became weak. The reason for this was not apparent until the aorta was decannulated; although the shape of the aorta was unchanged, a dissecting aneurysm was present. While the heart was massaged an external iliac artery was cannulated and bypass was reestablished. During replacement of the ascending aorta it was found that the right coronary artery had been completely transected and the distal end could not be found. Graft replacement of the ascending aorta was completed but the heart did not recover and the patient died on the table.

At necropsy the aorta showed extensive atheromatous changes. Clamping of the vessel caused separation of the diseased layer and blood was able to enter the potential space from the site of cannulation.

CASE 2 A woman aged 23 years had an atrial septal defect sutured under surface hypothermia in February 1958. Because of the large size of the defect there 1 Present address: Westminster Hospital, London, S.W.1 had been difficulty in closing the defect and two periods of inflow occlusion were needed. The patient remained well for seven years, then symptoms returned and increased during the next three years. Cardiac catheterization was performed in 1968 and it was demonstrated that the inferior vena cava had been diverted into the left atrium during the closure of the septal defect.

In August 1968, during routine cardiopulmonary bypass using the ascending aorta for arterial cannulation, the anomaly was corrected by reopening the atrial septum and inserting a Dacron patch, diverting the inferior vena caval blood into the right atrium.

Post-operative blood loss was excessive but the cardiovascular state remained excellent. Two weeks after the operation the patient became ill and developed a fever, and three days later the wound discharged pus. Staphyloccocus aureus sensitive to penicillin was isolated and with antibiotic treatment her general condition improved but she remained febrile. The wound continued to discharge. On the 25 th post-operative day the wound was reopened to improve drainage. Pus and fibrin clots were removed from behind the sternum and this was followed by torrential arterial bleeding. The sternum was opened, the clot from a false aneurysm evacuated, and the bleeding was controlled with a finger inserted into the hole in the ascending aorta. The left femoral artery and vein were cannulated, and bypass was established. The patient was cooled to $20^{\circ} \mathrm{C}$. At this temperature the circulation was arrested, the ascending aorta was mobilized, and a side clamp was applied. Circulation and rewarming were started while the aorta was closed by direct suture. In the post-operative period the patient remained febrile despite treatment with antibiotics. After 14 days antibiotics were stopped and a series of blood cultures were taken; all were negative. During this period her temperature settled and when she had been afebrile for four weeks the patient was discharged. She remained well until November 
1968, when she developed pain in the right chest. She was admitted to another hospital where positive blood cultures were obtained and she was then transferred to Shotley Bridge Hospital. Her general condition was good; she was febrile; there was a small fluctuating area in the centre of the wound, and the radiograph showed broadening of the right mediastinum.
Intravenous therapy with appropriate antibiotics was started. Four days later the superficial abscess was evacuated. Next day the false aneurysm ruptured and the patient exsanguinated in a few minutes.

At necropsy a large false aneurysm was present in the anterior mediastinum. The site of aortic cannulation had reopened (Fig. 1).
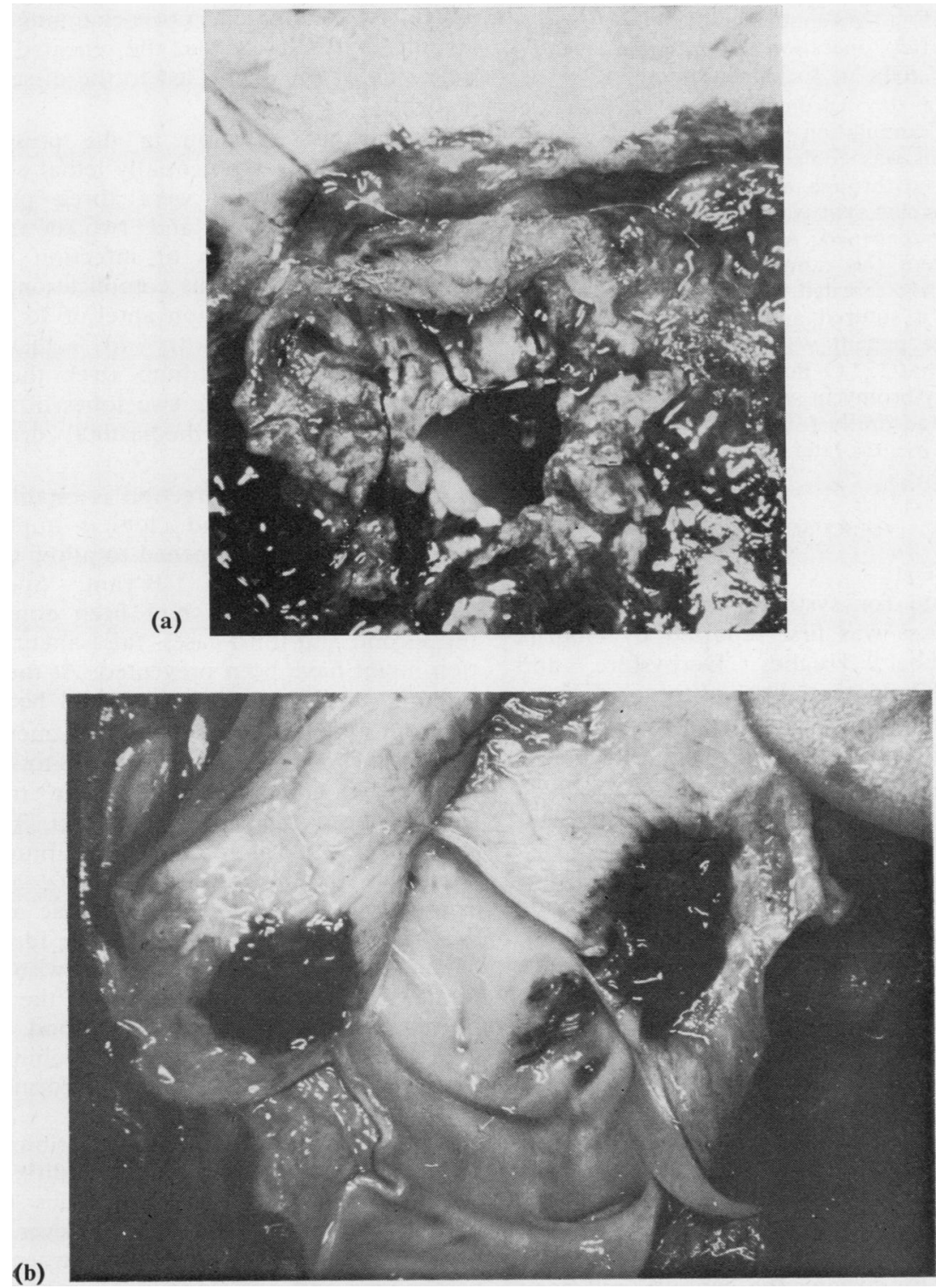

FIG. 1. (a) A view from the outside showing the hole in the aorta with clots all round. (b) $A$ view through the inside of the aorta showing a normal aortic wall, except at the edges of the defect, which were affected by infection. 
CASE 3 A 26-year-old woman had pulmonary stenosis. In February 1969 pulmonary valvotomy was performed using routine cardiopulmonary bypass with aortic cannulation.

She was febrile from the day of operation. A wound infection became evident on the 6 th post-operative day and the superficial part of the wound was opened and pus evacuated. Her temperature settled and on the 14th day secondary suture of the wound was performed. She ran a low-grade fever following this. Two months after operation a pulsating swelling appeared to the right of the mediastinum.

The following day cardiopulmonary bypass was established by cannulation of the femoral vessels. While the patient was cooled to $18^{\circ} \mathrm{C}$. the left ventricle was exposed through a small left thoracotomy and a left ventricular vent was inserted. At $20^{\circ} \mathrm{C}$. the sternotomy was reopened. A false aneurysm of the aorta arising from the cannulation site was found. The circulation was arrested, the aorta mobilized, and the opening in it sutured and covered with a pericardial flap. The patient was rewarmed and bypass was discontinued at $37^{\circ} \mathrm{C}$. Post-operatively cloxacillin. Fucidin, and erythromycin were given intravenously for four weeks and orally for four weeks. The patient remains well six months later.

\section{DISCUSSION}

The aortic route for systemic return in cardiopulmonary bypass was first reported by Dodrill, Marshall, Nyboer, Hughes, Derbyshire, and Stearns (1957). The potentialities of this route were soon realized and many techniques were described for ascending aortic cannulation (Nuñez and Baily, 1959 ; De Wall and Levy, 1963 ; McAlpine, Selman, and Kawakami, 1967; Gerbode, Kerth, Kovacs, Sanchez, and Hill, 1968).

Since October 1966 cannulation of the ascending aorta, by the method described by Gerbode et al. (1968), has been used routinely in this unit. This site of cannulation is preferred to the femoral or iliac artery because of its simplicity, convenience, and low complication rate.

Reviews of this method of cannulation (De Wall and Levy, 1963 ; Gerbode et al., 1968 ; McAlpine et al., 1967; Trimble and Bigelow, 1966) report a low incidence of complications when compared with the femoral route (Gerbode et al., 1968; McAlpine et al., 1967). Cerebral damage has been reported (Kulkarni, 1968) following direct perfusion into the left common carotid artery. This can be avoided by curving the cannula and confirming that the end is directed to the inferior aspect of the aorta before starting cardiopulmonary bypass.
Conklin believes that the chief benefit of this method is that it prevents retrograde aortic dissection (Conklin and Giannelli, 1966). Our experience suggests that an obviously atheromatous aorta may be an indication for femoral artery cannulation, although patients with severe atheroma are the ones at greater risk of dissection from femoral cannulation. If the atheromatous aorta is cannulated, cross-clamping should be avoided; it is likely that the repeated closing and releasing of the clamp led to the dissection in our patient.

Mediastinal infection in the presence of an aortic incision is a potentially lethal complication. In this series there were three patients with mediastinal infection and two developed false aneurysms. Prevention of infection is the ideal method of avoiding this complication. Prevention of haematoma formation anterior to the aorta is important. We now try to achieve this by resuturing the pericardium over the aorta, by reapproximation of the two lobes of the thymus, and by placing the mediastinal drainage tube anterior to the thymus.

Once mediastinal infection is established, early evacuation of pus and clots is important. The sternum should be reopened to allow débridement of the mediastinum (Bryant, Spencer, and Trinkle, 1969). If this had been done earlier in the second and third cases, false aneurysm formation might have been prevented. At the same time systemic antibiotic therapy should be started.

Deep hypothermia in the treatment of false aortic aneurysms was suggested to one of us (A. B.) by Ghadiali (1965). The method has recently been reported by Lillehei, Todd, Levy, and Ellis (1969), who used it for a ruptured mycotic aneurysm of the ascending aorta after aortic cannulation as well as other cardiac emergencies. We have no doubt that this is the ideal way out of an emergency which is otherwise invariably fatal. With the circulation arrested the wound can be reopened with little loss of blood, even when the aneurysm is entered, and mobilization and closure of the aorta can be performed without difficulty.

In the post-operative period antibiotic therapy should be as for bacterial endocarditis with intravenous followed by oral antibiotics for a total of six weeks after control of the fever. If there is no response to the antibiotic treatment it should be changed and not stopped. Discontinuing treatment too soon in case 2 may have led to inadequate control and later recrudescence of the infection. 


\section{REFERENCES}

Bryant, L. R., Spencer, F. C., and Trinkle, J. K. (1969). Treatment of median sternotomy infection by mediastinal irrigation with an antibiotic solution. Ann. Surg., 169, 914.

Conklin, E. F., and Giannelli, S., Jr. (1966). Arterial perfusion into the ascending aorta during cardiopulmonary bypass. Ann. thorac. Surg., 2, 858.

De Wall, R. A., and Levy, M. J. (1963). Direct cannulation of the ascending aorta for open-heart surgery. J. thorac. cardiovasc. Surg., 45, 496.

Dodrill, F. D., Marshall, N., Nyboer, J., Hughes, C. H., Derbyshire, A. J., and Stearns, A. B. (1957). The use of heart-lung apparatus in human cardiac surgery. $J$. thorac. Surg., 33, 60.

Gerbode, F., Kerth, W. J., Kovacs, G., Sanchez, P. A., and Hill, J. D. (1968). Cannulation of the ascending aorta for perfusion during cardiopulmonary bypass. A new technique and analysis of results. J. cardiovasc. Surg. (Torino), 9, 293.
Ghadiali, P. (1965). Personal communication.

Kulkarni, M. G. (1968). A complication of aortic cannulation. J. cardiovasc. Surg. (Torino), 9, 207.

Lillehei, C. W., Todd, D. B., Levy, M. J., and Ellis, R. J. (1969). Partial cardiopulmonary bypass, hypothermia, and total circulatory arrest. A lifesaving technique for ruptured mycotic aortic aneurysms, ruptured left ventricle, and other complicated cardiac pathology. J. thorac. cardiovasc. Surg., 58, 530.

McAlpine, W. A., Selman, M. W., and Kawakami, T. (1967). Routine use of aortic cannulation in open-heart operations. Experience with an improved technique. Amer. J. Surg., 114, 831.

Nuñez, L. E., and Baily, C. P. (1959). New method for systemic arterial perfusion in extracorporeal circulation. J. thorac. Surg., 37, 707.

Trimble, A. S., and Bigelow, W. G. (1966). Proximal aortic cannulation for extracorporeal circulation. J. thorac. cardiovasc. Surg. (Torino), 51, 674. 